\title{
An Approximate Semantic Model of Hybrid Systems
}

\author{
Shihan Yang \\ Guangxi Key Laboratory of Hybrid Computation and IC \\ Design Analysis, Nanning, China \\ School of Information Science and Engineering \\ Guangxi University of Nationalities \\ Nanning, China \\ Qiong Yu \\ School of Science \\ Guangxi University of Nationalities \\ Nanning, China \\ Guangxi Key Laboratory of Hybrid Computation and IC \\ Design Analysis \\ Nanning, China
}

\author{
Houwen Liu \\ Chengdu Aerospace Communication Device Company Li- \\ mited \\ Chengdu, China \\ Jinzhao Wu \\ Guangxi Key Laboratory of Hybrid Computation and IC \\ Design Analysis \\ Nanning, China \\ School of Science \\ Guangxi University of Nationalities \\ Nanning, China \\ School of Inf. Science and Eng., \\ Guangxi University of Nationalities \\ Nanning, China
}

\begin{abstract}
Hybrid system is a dynamic system. However, it is difficult to formally analyze hybrid systems due to the continuous parts. In this paper, we propose an approximate semantic model of hybrid systems, in order to employ formal analyzing techniques; furthermore, the error could be under control. This method is based on incomplete low-up matrix decomposition, which is used to generate approximating transition between states for the continuous components of hybrid systems. The technique reduces the complexity of analyzing computation. Moreover, the model is also used to approximating control the transition conditions, which simplifies the conditions.
\end{abstract}

Keywords-hybrid systems; approximate analyzing model; incomplete decomposition; error analysis

\section{INTRODUCTION}

Approximation of purely discrete systems has been based on language inclusion and equivalence with notions such as simulation or bisimulation relations [1].The notion of bisimulation has been instrumented in obtaining decidability results for various classes of hybrid systems[2].Notions that are similar to bisimulation have been considered in supervisory control of discrete event systems [3], and hybrid systems [4]. Bisimulations have also been used as a controller synthesis tool for discrete-event systems[5].However, these methods can only ensure the systems approximately satisfy the given rang and not be able to calculate the allowable error of transition between states.

The basic method of incomplete factorizations is used of incomplete LU decomposition. The most well-known is the incomplete triangular decomposition ILU0 [6]. However, the ILU preconditioner does not perform well for some PDE problems [7]. Thus, modified ILU (MILU) preconditioner is proposed [8].

In this work, we review the hybrid systems and its classical semantic model in section 2 . In section 3 , approximate tech- niques employed should be demonstrated. Then the approximate semantic model and its error analyzing are considered in detail in section 4 . Section 5 draws conclusion and proposes the future works.

\section{HYBRID SYSTEMS AND ITS SEMANTIC MODEL}

Definition 1 A hybrid system [9] is defined as a tuple

$$
H=\left(L, n, p, E, F, \operatorname{Inv}, G, R, Q^{\circ}\right), \text { where }
$$

- $L$ is a finite set of locations or discrete states. $|L|$ denotes the number of elements of $L, L=\{1, \cdots,|L|\}$.

- $n: L \rightarrow \mathrm{N}$, where for every $l \in L, n_{l}$ is the dimension ofthe continuous state space in the location $l$. The set of states of the hybrid system is $Q=\bigcup_{l \in L}\{l\} \times R^{n_{i}}$.

- $p: L \rightarrow \mathrm{N}$, where for every $l \in L, p_{1}$ is the dimension of the continuous observation of the hybrid system in the location $l$. The set of observations is $\Pi=\bigcup_{l=I}\{l\} \times R^{p_{1}}$.

- $E \subseteq L \times L$ is the set of events or discrete transitions.

- $F=\left\{F_{l} \mid l \in L\right\}$ defines the continuous dynamics in each location. For each $l \in L, F_{l}$ is a triple $\left(f_{1}, g_{l}, U_{l}\right)$ where $f_{l}$ : $R^{n_{l}} \times U_{l} \rightarrow R^{n_{l}}, g_{l}: R^{n_{l}} \rightarrow R^{p_{l}}$ and $U_{l} \subseteq R^{m_{l}}$ is a compact set of internal inputs accounting for disturbances and modeling uncertainties. While the discrete part of the state is $l$, the continuous part evolves according to

$$
\left\{\begin{array}{c}
x(t)=f_{l}(x(t), u(t)), u(t) \in U_{l} \\
y(t)=g_{l}(x(t)) .
\end{array}\right.
$$

- Inv $=\left\{\operatorname{Inv}_{l} \mid l \in L\right\}$ defines an invariant set in each location. For each $l \in L, \quad I n v_{l} \subseteq R^{n_{l}}$ constrains the value of the continuous part of the state while the discrete part is $l$. 
- $G=\left\{G_{e} \mid e \in E\right\}$ defines the guard for each discrete transition. For each $e=\left(l . l^{\prime}\right) \in E, G_{e} \subseteq I n v_{l}$. The discrete transition $e$ is enabled when the continuous part of the state is in $G_{e}$.

- $R=\left\{R_{e} \mid e \in E\right\}$ defines the reset map for each discrete transition. For each $e=\left(l . l^{\prime}\right) \in E, R_{e}: G_{e} \rightarrow 2^{l w_{i}}$. When the event $e$ occurs, the continuous part of the state is reset using $R_{e}$.

- $Q^{0} \subseteq Q$ is the set of initial states: $Q^{0}=\bigcup\{l\} \times I_{l}^{0}$, with $I_{l}^{0} \subseteq I n v_{l}$.

Here, we consider that hybrid systems can be formulated as transition systems [10]. The results can be reviewed in much detail in [11].

Definition 2 A labeled transition system with observations is a tuple $T=\left(Q, \Sigma, \rightarrow, Q^{0}, \Pi,\langle\langle\rangle\rangle\right)$ that consists of: a set $Q$ of states, a set $\Sigma$ of labels, a transition relation $\rightarrow \subseteq Q \times \Sigma \times Q$, a set $Q^{\circ} \subseteq Q$ of initial states, a set $\Pi$ of observations, and an observation map $\langle\langle\rangle\rangle:. Q \rightarrow \Pi$.

A state trajectory of $T$ is a sequence of transitions,

$$
q^{0} \stackrel{\sigma^{0}}{\longrightarrow} q^{1} \stackrel{\sigma^{1}}{\longrightarrow} q^{2} \stackrel{\sigma^{2}}{\longrightarrow} \cdots, \quad \text { where } q^{0} \in Q^{0} .
$$

Let $T_{1}=\left(Q_{1}, \Sigma_{1}, \rightarrow_{1}, Q_{1}^{0}, \Pi_{1},\langle\langle\cdot\rangle\rangle_{1}\right)$ and $T_{2}=\left(Q_{2}, \Sigma_{2}, \rightarrow_{2}, Q_{2}^{0}, \Pi_{2},\langle\langle\cdot\rangle\rangle_{2}\right)$ be two labeled transition systems with the same set of labels $\left(\Sigma_{1}=\Sigma_{2}=\Sigma\right)$ and the same set of observations $\left(\Pi_{1}=\Pi_{2}=\Pi\right)$ (i.e. $T_{1}$ and $T_{2}$ are elements of $T(\Sigma, \Pi)$ ). Let us assume that the set of observation $\Pi$ is a metric space; $d_{\Pi}$ denotes the metric of $\Pi$.

Definition 3 A relation $S_{\delta} \subseteq Q_{1} \times Q_{2}$ is a $\delta$-approximate simulation relation of $T_{1}$ by $T_{2}$ if for all $\left(q_{1}, q_{2}\right) \in S_{\delta}$ :

(1) $d_{\text {п }}\left(\left\langle\left\langle q_{1}\right\rangle\right\rangle_{1},\left\langle\left\langle q_{2}\right\rangle\right\rangle_{2}\right) \leq \delta$,

(2) $\forall q_{1} \stackrel{\sigma}{\longrightarrow} q_{1}^{\prime}, \exists q_{2} \stackrel{\sigma}{\longrightarrow} q_{2}^{\prime}$ such that $\left(q_{1}^{\prime}, q_{2}^{\prime}\right) \in S_{\delta}$.

Definition $4 T_{2}$ approximately simulates of $T_{1}$ with the precision $\delta$ ( noted $T_{1} \preceq_{\delta} T_{2}$ ), if there exists $S_{\delta}$, a $\delta$-approximate simulation relation of $T_{1}$ by $T_{2}$ such that for all $q_{1} \in Q_{1}^{0}$, there exists $q_{2} \in Q_{2}^{0}$ such that $\left(q_{1}, q_{2}\right) \in S_{\delta}$.

If $T_{2}$ approximately simulates $T_{1}$ with the precision $\delta$ then the language of $T_{1}$ is approximated with precision $\delta$ by the language of $T_{2}$.

Theorem 5 If $T_{1} \preceq_{\delta} T_{2}$, then for all external trajectories of $T_{1}$,

$$
\pi_{1}^{0} \stackrel{\sigma^{0}}{\longrightarrow} \pi_{1}^{1} \stackrel{\sigma^{1}}{\longrightarrow} \pi_{1}^{2} \stackrel{\sigma^{2}}{\longrightarrow} \cdots
$$

there exists an external trajectory of $T_{2}$ with the same sequence of labels

$$
\pi_{2}^{0} \stackrel{\sigma^{0}}{\longrightarrow} \pi_{2}^{1} \stackrel{\sigma^{1}}{\longrightarrow} \pi_{3}^{2} \stackrel{\sigma^{2}}{\longrightarrow} \cdots
$$

such that for all $i \in N, d_{\Pi}\left(\pi_{1}^{i}, \pi_{2}^{i}\right) \leq \delta[11]$. (Proof omitted.)

\section{APPROXIMATE AND INCOMPLETE FACTORIZATIONS}

The approximate solution for a large sparse linear system $A x=b$ is to find a matrix $M$ (the preconditioner) such that the original linear system is transformed into an equivalent linear system $M^{-1} A x=M^{-1} b$. Here, we focus on incomplete factorization preconditioners which are ILU and MILU. The basic idea of ILU preconditioner is to modify Gaussian elimination to allow fill-ins at only a restricted set of positions in the LU factors.

Let the allowable fill-in positions be given by the index set $S$, i.e.

(1) $l_{i, j}=0$ if $j>i$ or $(i, j) \notin S ; u_{i, j}=0$ if $i>j$ or $(i, j) \notin S$

A commonly used strategy is to define $S$ by:

(2) $S=\left\{(i, j) \mid a_{i, j} \neq 0\right\}$.

Let the preconditioner $M$ be defined by the product of the resulting $L U$ factors, i.e. $M=L U$.

(3) $m_{i, j}=a_{i, j}$ if $(i, j) \in S$.

The basic idea of MILU preconditioner is: in the condition (3) for ILU, the condition $m_{i, j}=a_{i, i}$ is removed and a new row sum condition is added. That is, (3) is replaced by:

(4) $\sum_{j=1}^{n} m_{i, j}=\sum_{j=1}^{n} a_{i, j} \forall i_{\text {and }} m_{i, j}=a_{i, j}$ if $i \neq j$ and $(i, j) \in S$.

Here, accuracy refers to the degree of preconditioner $M$ and matrix $A$, can be measured by the size of the $\|M-A\|_{F}$.

\section{Approximate Semantic Model}

Let $H=\left(L, n, p, E, F, \operatorname{Inv}, G, R, Q^{\circ}\right)$ be a hybrid system and $T=\left(Q, \Sigma, \rightarrow, Q^{0}, \Pi,\langle\langle\rangle\rangle\right)$ be the associated transition system. Here, we only consider the continuous state space in the location

$l \in L$ and the relation of states transition is $x=A x$. For, the continuous dynamic behavior $q \in Q_{\text {is abstracted into a plurality }}$ of continuous dynamic behaviors $\cdots q_{1} q_{2} q_{3} \cdots$, and every $q_{i}(i \in N)$, where $N$ is the set of positive integers, has the form of changing rules $x=A x$. For $t \in R^{+}$, where $R^{+}$is the set of positive real numbers, the continuous dynamic behavior trajectory is abstracted into:

$$
\cdots q_{1} \stackrel{\sigma^{1}}{\longrightarrow} q_{2} \stackrel{\sigma^{2}}{\longrightarrow} q_{3} \stackrel{\sigma^{3}}{\longrightarrow} \cdots
$$

and the mathematical relationship of $q_{1} \stackrel{\sigma^{1}}{\longrightarrow} q_{2}, q_{2} \stackrel{\sigma^{2}}{\longrightarrow} q_{3}$ is respective $x=A_{1} x, y=A_{2} y$. Thus, a transition from behavior $q_{1}$ to behavior $q_{3}$ can be expressed as $q_{1} \stackrel{\sigma^{2} \sigma^{1}}{\longrightarrow} q_{3}$.

Consider the continuous dynamic behavior $q_{1} \stackrel{\sigma^{1}}{\longrightarrow} q_{2}$ is following as: 


$$
\left\{\begin{array}{c}
x_{1}=a_{11}(t) x_{1}+a_{12}(t) x_{2}+\cdots+a_{1 n}(t) x_{n} \\
x_{2}=a_{21}(t) x_{1}+a_{22}(t) x_{2}+\cdots+a_{2 n}(t) x_{n} \\
\cdots \cdots \\
x_{n}=a_{n 1}(t) x_{1}+a_{n 2}(t) x_{2}+\cdots+a_{n n}(t) x_{n}, t \in\left[t_{1}, t_{2}\right] .
\end{array}\right.
$$

And the initial behavior is $x\left(t_{1}\right)=\left(b_{1}, b_{2}, \cdots, b_{n}\right)$.

It can be expressed as: $x=A(t) x, t \in\left[t_{1}, t_{2}\right]$,

where

$$
A(t)=\left[\begin{array}{cccc}
a_{11}(t) & a_{12}(t) & \cdots & a_{1 n}(t) \\
a_{21}(t) & a_{22}(t) & \cdots & a_{2 n}(t) \\
\vdots & \vdots & & \vdots \\
a_{n 1}(t) & a_{n 2}(t) & \cdots & a_{n n}(t)
\end{array}\right], \quad X=\left[\begin{array}{c}
x_{1} \\
x_{2} \\
\vdots \\
x_{n}
\end{array}\right], \quad x=\left[\begin{array}{c}
x_{1} \\
x_{2} \\
\vdots \\
x_{n}
\end{array}\right] .
$$

The original system $x=A(t) x$ is transformed into an equivalent system $M^{-1} A x=M^{-1} x$ by the mean of ILU, where $M=L U$ is the preconditioner, $L$ is a triangular matrix, $U$ is an upper triangular matrix. Because the system $x=A(t) x$ is a portrait of the transition process from $q_{1}$ to $q_{2} \cdot A-M$, an error matrix, has the meaning that $q_{1}$ can be translated into $q_{2}$ in the error, and its degree of approximation is $\|M-A\|_{F}$.

Definition 6 The approximate distance between $q_{1}$ and $q_{2}$ as $d\left(q_{1}, q_{2}\right)=\|M-A\|_{F}$.

We can call $d\left(q_{1}, q_{2}\right)=\|M-A\|_{F}$ as the degree of approximation of $q_{1}$ translated into $q_{2}$. Because $x\left(t_{1}\right)=q_{1}, x\left(t_{2}\right)=q_{2}$, the solution $y$ of the equivalent system $M^{-1} A x=M^{-1} x$ is the approximate solution of the original system $x=A(t) x$, where $y\left(t_{1}\right)=q_{1}, y\left(t_{2}\right)=q_{2}^{\prime}$. The mathematical relationship of $q_{1} \stackrel{\sigma}{\longrightarrow} q_{2}^{\prime}$ is: $y=A(t) y, t \in\left[t_{1}, t_{2}\right]$,

where $y=\left(\begin{array}{llll}y_{1} & y_{2} & \cdots & y_{n}\end{array}\right)^{T}, y=\left(\begin{array}{llll}y_{1} & y_{2} & \cdots & y_{n}\end{array}\right)^{T}$.

And the initial state is $y\left(t_{1}\right)=\left(b_{1}, b_{2}, \cdots, b_{n}\right)$.

Therefore, the accuracy between exact solution $X$ and approximate solution $y_{\text {is }} \delta=\|M-A\|_{F}$.

Theorem 7Let $y$ be the approximate solution of $x=A(t) x$, then $y=A(t) y$ simulates approximately to $x=A(t) x$ with precision $\lim _{t \rightarrow t_{2}}\|A(t)\|_{F}\|M-A\|_{F}$.

$$
\text { Proof: } \begin{aligned}
\lim _{t \rightarrow t_{2}}\|x-y\|_{F} & =\lim _{t \rightarrow t_{2}}\|A(t) x-A(t) y\|_{F}=\lim _{t \rightarrow t_{2}}\|A(t)(x-y)\|_{F} \\
& \leq \lim _{t \rightarrow t_{2}}\|A(t)\|_{F}\|x-y\|_{F} \leq \lim _{t \rightarrow t_{2}}\|A(t)\|_{F} \lim _{t \rightarrow t_{2}}\|x-y\|_{F} \\
& =\lim _{t \rightarrow t_{2}}\|A(t)\|_{F}\|M-A\|_{F}
\end{aligned}
$$

Hence, we can say the continuous dynamic behavior $q_{2}$ can be approximately simulated by $q_{2}^{\prime}$.

For location $l \in L$, the continuous dynamic behavior $q$ is abstracted into a plurality of continuous dynamic behaviors $\cdots q_{1} q_{2} q_{3} \cdots, q$ is approximately simulated into $q^{\prime}$, that is $\cdots q_{1}^{\prime} q_{2}^{\prime} q_{3}^{\prime} \cdots$. This means the set of continuous dynamic behavior $\cdots q_{1}^{\prime} q_{2}^{\prime} q_{3}^{\prime} \cdots$ can be abstracted into a continuous behavior $q^{\prime}$

Here, we mainly focus on the discrete switching between different discrete states. The continuous dynamic state $q$ in the location $l \in L$ is abstracted into a set of continuous dynamic states $\cdots q_{1} q_{2} q_{3} \cdots$, then the set of continuous dynamic states $\cdots q_{1} q_{2} q_{3} \cdots$ are approximately simulated to $\cdots q_{1}^{\prime} q_{2}^{\prime} q_{3}^{\prime}$ via the ILU method. That is, $l \stackrel{\tau}{\longrightarrow} l^{\prime}\left(\operatorname{iff}\left(l, l^{\prime}\right)=e \in E\right)$ becomes $\cdots q_{1}^{\prime} q_{2}^{\prime} q_{3}^{\prime} \cdots \stackrel{\tau}{\longrightarrow} l^{\prime}$, here, $\tau$ is a discrete transition.

We discuss the discrete transition $\tau$ (condition). Its form likes this: $g_{i}\left(x_{1}, x_{2}, \cdots, x_{n}\right)=a_{i 1} x_{1}+a_{i 2} x_{2}+\cdots+a_{i n} x_{n}(i=1,2, \cdots, n)$.

We only study the form of $g_{i}\left(x_{1}, x_{2}, \cdots, x_{n}\right)$. Sometimes, all of variables are difficult to satisfy the scope of $g_{i}\left(x_{1}, x_{2}, \cdots, x_{n}\right)$. Thus we can simplify the condition. We put all of the condition considered. It likes this:

$$
\left\{\begin{aligned}
g_{1}\left(x_{1}, x_{2}, \cdots, x_{n}\right)= & a_{11} x_{1}+a_{12} x_{2}+\cdots+a_{n n} x_{n} \\
g_{2}\left(x_{1}, x_{2}, \cdots, x_{n}\right)= & a_{21} x_{1}+a_{22} x_{2}+\cdots+a_{2 n} x_{n} \\
& \cdots \\
g_{n}\left(x_{1}, x_{2}, \cdots, x_{n}\right)= & a_{n 1} x_{1}+a_{n 2} x_{2}+\cdots+a_{n n} x_{n}
\end{aligned}\right.
$$

Here, let

$$
A=\left[\begin{array}{cccc}
a_{11} & a_{12} & \cdots & a_{1 n} \\
a_{21} & a_{22} & \cdots & a_{2 n} \\
\vdots & \vdots & & \vdots \\
a_{n 1} & a_{n 2} & \cdots & a_{n n}
\end{array}\right], \quad x^{\prime}=\left[\begin{array}{c}
g_{1}\left(x_{1}, x_{2}, \cdots, x_{n}\right) \\
g_{2}\left(x_{1}, x_{2}, \cdots, x_{n}\right) \\
\vdots \\
g_{n}\left(x_{1}, x_{2}, \cdots, x_{n}\right)
\end{array}\right], \quad X=\left[\begin{array}{c}
x_{1} \\
x_{2} \\
\vdots \\
x_{n}
\end{array}\right] .
$$

Thus (1) is equivalent to $x^{\prime}=A x$. We can obtain the preconditioner matrix $M$ of matrix $A$ by the mean of ILU. We assume that the form of $M$ likes this:

$$
M=\left[\begin{array}{cccc}
b_{11} & b_{12} & \cdots & b_{1 n} \\
b_{21} & b_{22} & \cdots & b_{2 n} \\
\vdots & \vdots & & \vdots \\
b_{n 1} & b_{n 2} & \cdots & b_{n n}
\end{array}\right] .
$$

Then $M$ replaces $A$ in the equation $x^{\prime}=A x$, thereby we can obtain equations like this:

$$
\left\{\begin{array}{c}
g_{1}\left(x_{1}, x_{2}, \cdots, x_{n}\right)=b_{11} x_{1}+b_{12} x_{2}+\cdots+b_{1 n} x_{n} \\
g_{2}\left(x_{1}, x_{2}, \cdots, x_{n}\right)=b_{21} x_{1}+b_{22} x_{2}+\cdots+b_{2 n} x_{n} \\
\vdots \\
g_{n}\left(x_{1}, x_{2}, \cdots, x_{n}\right)=b_{n 1} x_{1}+b_{n 2} x_{2}+\cdots+b_{n n} x_{n}
\end{array}\right.
$$

The values of variables in (1) are different from that of (2) due to the preconditioner $M$. It's difficult to control the precise conditions for states transitions. So we only find the approximating conditions for states transitions. 
Error of exact solution for (1) is $\|A-M\|_{F}$. We assume that the precise conditions is $\eta$, so the range of variables only belongs to $\left[-\|A-M\|_{F}+\eta,\|A-M\|_{F}+\eta\right]$,there states transition can come true. Here, states transition only is approximation.

\section{CONCLUSION}

A new modeling of hybrid systems is proposed by means of incomplete low-up matrix decomposition. Extending hybrid systems' semantic models to approximation semantic models enables the formal techniques to analyze hybrid systems. In addition, we analyze the error in detail, which could be under control according to proper decomposition condition.

\section{ACKNOWLEDGEMENTS}

This work is supported by the National Natural Science Foundation of China under Grant No.11371003 and No.61420009, the Natural Science Foundation of Guangxi under Grant No.2014GXNSFAA118359, No. 2012GXNSFGA060003 and No.2014GXNSFAA118358, the Science and Technology Foundation of Guangxi under Grant No.10169-1, and the Scientific Research Project No. 201012MS274 from Guangxi Education Department. And we thank reviewers very much for their careful reading and valuable comments that help us to improve the paper dramatically.

\section{REFERENCES}

[1] E.M.Clarke, O.Grumberg, and D.A.Peled.Model checking.MIT Press, 2000.

[2] Alur, R.,Henzinger, T., Lafferriere, G., \& Pappas, G. J. Discrete abstractions of hybrid systems. Proceedings of the IEEE, 88(7),971-984, 2000.

[3] A.Maleki, A. Askarzadeh. Comparative study of artificial intelligence techniques for sizing of a hydrogen-based standalone photovoltaic/wind hybrid system.International Journal of Hydrogen Energy, 2014, 39 (19).

[4] Lordache, Marian V, Antsaklis, Panos J. DES abstractions for the supervisory control of hybrid systems. Transactions of the Institute of Measurement and Control, Vol. 32 (5), pp. 468-486, 2010.

[5] Antoine Girard. Controller synthesis for safety and reachability via approximate bisimulation.Automatica, Vol. 48 (5), pp. 947-953, 2012.

[6] A. ElMaliki, R. Guénette. Efficient preconditioning techniques for finite element quadratic discretization arising from linearized incompressible Navier-Stokes equations. Int. J. Numer. Meth.Fluids, 2010, Vol. 63 (12).

[7] Ana Amal, Femando Casas. Exponential polar factorization of the fundamental matrix of linear differential systems.Journal of Computational and Applied Mathematics, 2013.

[8] T. Gustafsson. A class of first-order factorization methods. BIT, 18(1978), 142-156.

[9] A.Benveniste, T. Bourke, B.Caillaud, M.Pouzet. Nonstandard semantics of hybrid systems modelers.J. of Computer and System Sciences, 2012, Vol. 78 (3), pp.877-910.

[10] LIN Wang, WU Min, YANG ZhengFeng, ZENG ZhenBing. Exact safety verification of hybrid systems using sums-of squares representation. SCIENCE CHINA Information Sciences, 2014, 57:052101(13).

[11] Girard A, Pappas G J. Approximation metrics for discrete and continuous systems[J]. IEEE Transactions on Automatic Control, 2005, 52(5):782--798. 